Revue de l'Institut des langues et cultures

d'Europe, Amérique, Afrique, Asie et Australie

$21 \mid 2015$

Discours politique et culturel dans la Russie

contemporaine

La persistance du soviétique dans le discours

politique des dirigeants de la Russie contemporaine sur les technologies de l'information et de la communication

The Persistence of the Soviet Elements in the Political Discourse of Contemporary Russian Leaders on Information and Communications Technology (ICT)

Alexander Kondratov

CpenEdition

Journals

Édition électronique

URL : http://journals.openedition.org/ilcea/3035

DOI : 10.4000/ilcea.3035

ISSN : 2101-0609

Éditeur

UGA Éditions/Université Grenoble Alpes

Édition imprimée

ISBN : 978-2-84310-294-3

ISSN : 1639-6073

Référence électronique

Alexander Kondratov, «La persistance du soviétique dans le discours politique des dirigeants de la Russie contemporaine sur les technologies de l'information et de la communication », ILCEA [En ligne], 21 | 2015, mis en ligne le 01 février 2015, consulté le 01 mai 2019. URL : http:// journals.openedition.org/ilcea/3035; DOI : 10.4000/ilcea.3035

Ce document a été généré automatiquement le 1 mai 2019.

(C) ILCEA 


\section{La persistance du soviétique dans le discours politique des dirigeants de la Russie contemporaine sur les technologies de l'information et de la communication}

The Persistence of the Soviet Elements in the Political Discourse of Contemporary Russian Leaders on Information and Communications Technology (ICT)

\section{Alexander Kondratov}

Actuellement, en Russie post-soviétique, nous observons un paradoxe remarquable. Malgré la libéralisation du pays de l'ordre soviétique, installation des institutions démocratiques et la promesse de la liberté d'expression, nous remarquons l'absence de cette liberté et le retour du système de contrôle social complexe. L'État s'immisce à nouveau dans tous les domaines de la vie sociale en tant qu'acteur et régulateur principal. Les médias de masse sont soumis au contrôle complexe et à la censure, les institutions non lucratives sont instrumentalisées par l'État. Dans ces conditions, en Russie il y a un fort ancrage social des dispositifs numériques de communication. En 2013, le pays occupe la première place en Europe d'après le nombre d'utilisateurs de l'Internet. Selon les statistiques de l'institut de sondage TNS Sofres, $85 \%$ d'internautes russes ont un profil dans un réseau social ou le consultent régulièrement. $70 \%$ des utilisateurs d'Internet consultent des blogs au moins une fois par jour et $34 \%$ possèdent leurs propres blogs. Ces outils de communication numériques sont censés d'avoir une forte capacité de libéralisation et d'échanges en dehors de structures contrôlées par l'État post-soviétique. Nous considérons intéressant de s'interroger sur la place attribuée à ces technologies de l'information et de la communication (TIC) dans le discours politique russe contemporain. Notre souhait est d'étudier les enjeux de ces discours politiques aux éléments soviétiques qui traitent les technologies numériques. Quels moyens discursifs sont mobilisés ? Quelles figures rhétoriques et quel imaginaire les leaders post-soviétiques mobilisent pour traiter 
ce sujet? Quelle place occupe cet objet dans leurs stratégies discursives? D'après l'hypothèse que nous développons dans cet article, ce discours "soviétique » sur les nouvelles technologies joue un rôle très important dans la légitimation du régime politique russe contemporain.

\section{Cadrage théorique}

2 Les études du discours officiel soviétique sont nombreuses en Occident, notamment en France. Nous proposons de regrouper ces recherches en trois courants. Le premier, une approche de «soviétologie », représentée par Alex Inkeles, Peter Kenez et David Benn, considère que le discours du type soviétique n'est pas une forme particulière du discours politique. Il s'agit de l'ensemble des moyens de persuasion mobilisé par l'appareil étatique soviétique pour une diffusion plus efficace des messages officiels, voire la propagande (Inkeles, 1963 ; Kenez, 1985).

3 Une deuxième approche, dans les années 1970, propose d'étudier la "langue de bois ${ }^{1}$ » officielle soviétique comme un objet particulier de recherches. Michel Heller considère le langage officiel soviétique comme une des formes d'oppression. Ce langage détourne la réalité et fait appel aux émotions plutôt qu'aux faits. La principale caractéristique de cette langue, selon Heller, c'est son caractère artificiel et planifié. L'État soviétique avait le pouvoir de décider de la signification des mots et du contexte où ils doivent être employés :

La langue soviétique est un sous-produit de la vraie langue russe, nationalisée et trafiquée par un pouvoir soviétique qui en aurait fait son monopole. Les procédés seraient la censure du lexique et la réduction de la syntaxe à une construction du type slogan (forme simple et contenu primitif). (Heller, $1984: 276$ )

Heller considérait les citoyens soviétiques comme bilingues, car ils choisissaient le langage officiel soviétique ou la langue russe vivante selon les circonstances, dans le cercle familial ou à une réunion politique, par exemple. En poursuivant ces réflexions, Alain Besançon a proposé le concept de logocratie. Ce chercheur voyait la langue soviétique comme un des outils de la domination du régime: «Dans ce régime où le pouvoir est "au bout de la langue", l'indice d'extension de la langue de bois est l'indice le plus sûr d'extension du pouvoir. » (Besançon, 1980 : 206)

4 Une troisième approche a été développée par les linguistes à l'université de Grenoble au début des années 1980. Leurs idées étaient exposées dans la revue scientifique Essais sur le discours soviétique. Ce groupe considérait que le concept de la langue de bois réduit la langue uniquement à sa dimension idéologique et propose d'analyser les discours politiques russes par une analyse linguistique. L'ouvrage phare de ce groupe, intitulé Analyse du discours politique soviétique, a vu le jour en 1985 sous la direction de Patrick Sériot. Ce chercheur proposait d'étudier le fonctionnement du discours politique soviétique dans sa matérialité et étudier comment ce type de discours employait la langue russe. Cette langue du pouvoir officiel soviétique manifeste le lien explicite entre l'exercice du pouvoir politique et les pratiques langagières. Cette langue établit des relations avec les objets préconstruits et non avec les faits. Ce type de discours politique représentait le phénomène unique linguistique et géographiquement localisé. Il est caractérisé, comme Patrick Sériot l'indique, par la stratification hiérarchisée de plusieurs espaces énonciatifs où les « voix » ne sont pas égales. La caractéristique principale de ce discours c'est « une tension extrême entre une homogénéité, un monolithisme déclaré et 
une hétérogénéité fondamentale. Cette hétérogénéité, qu'on peut répéter dans des phénomènes profonds de la syntaxe, manifeste la présence constante de discours adverses et antérieurs » (Sériot, $1986: 31$ ).

Après la chute de l'URSS en 1991, l'État russe post-soviétique a déclaré la rupture avec le passé soviétique autoritaire. Par conséquent, les recherches sur le discours soviétique se sont graduellement estompées. Pourtant, une chercheuse britannique, spécialiste de la politique soviétique, Mary McAuley, considère que l'objet de recherches existe toujours et que les recherches ont été arrêtées prématurément. La période post-soviétique n'a pas créé de nouveau langage politique. La persistance du langage politique soviétique, selon McAuley, peut être une des explications de l'échec des réformes politiques et le retour rapide des facteurs soviétiques (McAuley, 1997).

Depuis quelques années, la présence du soviétique dans les discours politiques a de nouveau intéressé les chercheurs. Des conférences ont été organisées dans les universités françaises pour réfléchir sur le retour du « soviétique ». Par exemple, du 6 au 7 décembre 2012 les journées d'études «Le discours politique russe contemporain: aspects linguistiques, sociologiques et culturels » ont été organisées par l'Institut des langues et cultures d'Europe, Amérique, Afrique, Asie, Australie à l'université Stendhal-Grenoble 3.

\section{Étude qualitative du corpus}

7 Afin de réaliser notre recherche, nous avons construit notre premier corpus à partir de 16 discours de Vladimir Poutine et de 31 discours de Dmitri Medvedev prononcés entre 2000 et $2012^{2}$. Ce nombre de discours a été établi après avoir éliminé les discours doublons qui abordent les mêmes sujets, les discours qui ne représentent pas d'intérêt pour l'analyse (lettres, félicitations officielles) et les discours qui ne révèlent pas les stratégies des pouvoirs russes par rapport aux NTIC et l'Internet. Ainsi s'agit-il de textes homogènes marqués produits dans des conditions identiques dans le cadre des mêmes institutions. Pour notre analyse quantitative nous avons utilisé un logiciel d'analyse qualitative QDA Miner version 4.1.8.

Nous avons classé ces discours en trois types:

- le premier type est composé des discours adressés au grand public, à l'ensemble des citoyens du pays. Ce type de discours prononcés dans le cadre de la communication politique sert à définir les grandes lignes politiques et idéologiques du régime russe contemporain aux niveaux national et international. Les pouvoirs publics mobilisent de nombreux dispositifs, tels que des conférences de presse nationales, des "Lignes directes", des articles de journaux. Ces discours ont une large couverture par les médias dominants, surtout par les chaînes télévisées généralistes (Perviy Kanal, chaîne Rossia et NTV) ;

- le deuxième type inclut des discours adressés à l'élite politique, économique ou intellectuelle de la Russie ou aux fonctionnaires au sens large. Ils sont considérés par les élites politiques comme un « agenda et programme politique et économique » (Zorkin, 2011) du pays et ont une grande influence sur l'opinion publique. Ce sont des discours annuels rituels devant l'Assemblée fédérale, des discours devant les réunions du Conseil d'État et lors des forums économiques nationaux et internationaux;

- le troisième type de discours s'adresse à différentes catégories socioprofessionnelles plus restreintes (réunions avec les ministres, rencontres avec des étudiants, des journalistes, des 
chercheurs). Ces discours visent un double objectif. Ils développent les propos des autres discours et en même temps tiennent à séduire d'autres groupes sociaux.

La classification des discours sur NTIC et Internet est présentée dans le tableau qui suit.

Tableau 1. - La classification des discours sur Internet et TIC par rapport au public visé.

\begin{tabular}{|c|c|c|}
\hline TYPES & Poutine & Medvedev \\
\hline & discours choisis & 31 discours choisis \\
\hline Type 1 & 7 & 4 \\
\hline Type 2 & 5 & 7 \\
\hline Type 3 & 4 & 20 \\
\hline
\end{tabular}

10 Cette classification des discours nous permet de tirer nos premières conclusions. La distribution de discours sur les NTIC entre ces trois types montre que les discours de Poutine et de Medvedev se complètent. En effet, Vladimir Poutine s'adresse à l'audimat national avec la stratégie de légitimer sa figure symbolique en tant que "père de la Nation ». Dmitri Medvedev, au contraire, cherche à faire adhérer des groupes sociaux qui échappent à la propagande étatique, tels que l'intelligentsia, des scientifiques, des élites techniques et financières, des journalistes qui ont adopté une attitude critique concernant les discours de Poutine. Nous observons que ces hommes politiques mènent un jeu assez subtil dans l'optique d'adapter leurs discours au public visé. Dans ces discours sur les NTIC, ils changent l'argumentation, la tonalité, les termes associés, les métaphores, le champ lexical des mots en fonction de la cible visée.

11 L'analyse des discours choisis montre que l'Internet et NTIC se trouvent à la marge des thèmes principaux des discours officiels. Dans ses huit discours devant l'Assemblée fédérale russe (AFR), Vladimir Poutine utilise le terme Internet trois fois sur 48143 mots, tandis que le terme État y apparaît 416 fois, le terme pouvoir 163 fois. Dans ses quatre discours devant l'AFR, Dmitri Medvedev utilise le mot Internet 14 fois sur 32259 mots, le mot État 207 fois et pouvoir 69 fois. Le premier discours de Vladimir Poutine sur les nouvelles technologies date de l'année 2001, un an après son arrivée au pouvoir. Le dernier discours étudié date du mois de décembre 2012. C'est sa $9^{\mathrm{e}}$ communication devant l'Assemblée. Les discours sélectionnés de Dmitri Medvedev ont été prononcés entre mars 2008 et mars 2012 lors de son mandat présidentiel. Tous les textes étudiés sont accessibles sur les sites officiels de ces deux hommes politiques ainsi que dans l'archive en ligne du journal quotidien officiel Rossiskaïa Gazeta ${ }^{3}$ et les sites des agences de presse russe (Interfax, Ria Novosti).

12 Notre deuxième corpus, constitué de 18 discours, est construit à partir des discours des autres hauts fonctionnaires d'État (chefs des départements, ministres, responsables politiques) prononcés à la même période. Nous considérons que les discours de ce type ont des relations complexes et entrelacées. Ils se complètent pour renforcer l'idée déjà exprimée par les dirigeants du pays. Ces discours peuvent même contredire les discours de premières personnes d'État. Dans ce dernier cas, ces discours des fonctionnaires servent à élargir la marge de manœuvre, notamment, au sujet du contrôle d'Internet. Par exemple, les fonctionnaires prônaient la censure sur Internet, tandis que le président s'abstenait de donner son avis à ce sujet en se réservant le rôle de l'arbitre. La fréquence des termes associés avec NTIC dans les discours de Poutine et Medvedev est présentée 
dans le tableau $\mathrm{n}^{\circ} 2$.

Tableau 2. - La fréquence des termes dans les discours de Poutine et Medvedev devant l'Assemblée fédérale de la Fédération de Russie entre 2001 et 2012.

\begin{tabular}{|l|l|l|}
\hline & POUTINE & MEDVEDEV \\
\hline Unité lexicale & 8 discours (48143 mots) & 4 discours (32 259 mots) \\
\hline « ИНТЕРНЕТ*» & $0,06 \%$ (29 fois) & $0,043 \%$ (14 fois) \\
\hline « НОВ* ТЕХНОлоги* & $0,091 \%(44)$ & $0,06 \%(19)$ \\
\hline « ИНФОРМАЦИОН*» & $0,027 \%(13)$ & $0,030 \%(10)$ \\
\hline « МОДЕРНИЗАЦИ* & $0,06 \%(28)$ & $0,037 \%(12)$ \\
\hline « ГОСУДАРСТВО » & $0,86 \%(416)$ & $0,64 \%(207)$ \\
\hline « ВЛАСТ* & $0,34 \%(163)$ & $0,213 \%(69)$ \\
\hline
\end{tabular}

L'analyse de la fréquence des mots et des termes utilisés dans les discours de Vladimir Poutine et Dmitri Medvedev nous amène à une première conclusion. Nous découvrons une ressemblance significative de ces discours. Cette ressemblance se manifeste par l'emploi des mots appartenant aux mêmes catégories. Nous rencontrons les mêmes mots avec la même fréquence dans les discours des deux présidents russes devant l'Assemblée fédérale. Par exemple, l'expression «нов* тЕхнологи*4 " (nouvelles technologies) apparait avec quasiment la même fréquence dans les discours de ces hommes politiques. Les termes « РОссия » (Russie), «ВЛАСТЬ » (pouvoir), « ЗАКон » (loi), « ГОсУДАРство » (État) И «ТРАДИЦИонн*» (traditionnel) sont les termes les plus fréquents dans les discours de ces deux hommes politiques. Cette analyse du vocabulaire des deux dirigeants post-soviétiques montre que malgré la différence proclamée entre leurs politiques, ces hommes politiques partagent un imaginaire politique commun.

\section{L'abondance du « soviétique » dans les discours politiques de Poutine}

Dans l'analyse des discours choisis, nous nous sommes appuyés sur les concepts de l'imaginaire socio-discursif proposés par le chercheur français Patrick Charaudeau et de l'imaginaire social introduit par le philosophe et économiste français Cornelius Castoriadis. Ce dernier définit l'imaginaire social comme "un univers de signification fondateur de l'identité du groupe dans la mesure où il est ce qui tient une société ensemble " (Castoriadis, 1975). Patrick Charaudeau considère que dans le discours politique, ces imaginaires sont soutenus par une rationalisation discursive et sont instrumentalisés à des fins de persuasion. L'auteur établit la typologie des imaginaires présents dans la plupart des discours politiques. Il s'agit, par exemple, de l'imaginaire de la «tradition», de la «modernité », de la « souveraineté populaire » (Charaudeau, 2005). 
Notre analyse montre que certains de ces imaginaires sont aussi présents dans les discours des dirigeants russes au même niveau que des imaginaires socio-discursifs propres aux discours politiques russes post-soviétiques.

L'imaginaire socio-discursif soviétique est traduit par des appels réguliers au passé soviétique en tant qu'élément de rhétorique. Dans cet imaginaire, la construction de l'identité de la Russie post-soviétique se base sur les valeurs et les expériences acquises pendant la période de l'existence de l'URSS. De l'autre côté, cet imaginaire peut se construire également par le rejet de ces valeurs soviétiques. Dans les deux cas, le passé soviétique sert de repère important pour la construction de la Russie actuelle. Politologue et historien de l'université d'État de Saint-Pétersbourg, Viatcheslav Morozov montre que le passé soviétique est un élément fondateur du projet de l'État russe contemporain. La période de l'existence de l'URSS est présentée comme le "siècle d'or de l'État russe " (МОРОзОВ, 2006). Les discours sur les nouvelles technologies ressemblent souvent à des rapports officiels soviétiques sur le progrès de l'économie :

ВЫСОКИМИ ТЕМПАМИ РАЗВИВАЛИСЬ ИНФОРМАЦИОННЫЕ ТЕХНОЛОГИИ. ТАК НАЗЫВАЕМАЯ НОВАЯ ЭКОНОМИКА У НАС РАСТЕТ. ПРИРОСТ ИХ ПРОДУКЦИИ СОСТАВЛЯЛ ОТ 20 ДО 30 ПРОЦЕНТОВ В ГОД. ЗНАЧИТЕЛЬНО ВЫРОС УРОВЕНЬ ТЕЛЕФОНИЗАЦИИ СТРАНЫ. ЧИСЛО ПОЛЬЗОВАТЕЛЕЙ МОБИЛЬНЫХ ТЕЛЕФОННЫХ АППАРАТОВ ЕЖЕГОДНО УДВАИВАЛОСЬ И ДОСТИГЛО ПОЧТИ 18 МИЛЛИОНОВ. ПО ОЦЕНКАМ, СЕГОДНЯ ОКОЛО 10 МИЛЛИОНОВ ЧЕЛОВЕК В РОССИИ ПОЛЬЗУЮТСЯ ИНТЕРНЕТОМ ${ }^{5}$.

Dans cet exemple, nous trouvons des caractéristiques propres à la "langue soviétique ». Notamment, il s'agit de l'abondance des noms, le recours aux tournures impersonnelles, l'emploi fréquent du comparatif. « Pour ce style, il n'y a pas de différence entre l'écrit et l'oral, ce discours s'adapte peu à son public, il ne croit pas nécessaire de prouver.» (Thom, 1987) Dans ses discours sur les NTIC, Poutine identifie bien la distance entre lui (l'orateur) et son public. Sa façon de parler est froide et succincte. Ceci est censé mettre en valeur sa position supérieure dans la hiérarchie de l'État russe et souligner la grandeur de sa figure symbolique qui est la source de stabilité et de protection. En même temps, le président utilise les éléments rhétoriques adaptés au public pour se rapprocher de son audience, comme, par exemple, anecdotes ou blagues populaires.

Les discours politiques des dirigeants russes s'adaptent aux événements et aux stratégies de la communication politique d'État. Nous avons étudié les discours de Vladimir Poutine du point de vue diachronique ayant défini trois périodes dans ses discours sur Internet et les nouvelles technologies. La première période commence en janvier 2000 avec l'arrivée de Poutine au pouvoir et se termine en 2004 à la fin de son premier mandat. Ses discours sont prononcés dans le contexte de l'établissement du système de contrôle social avec l'appui sur la bureaucratie. Au début de son premier mandat, Vladimir Poutine considère l'Internet dont l'audience en Russie n'est pas encore significative, comme un des dispositifs de communication qui n'est pas encore contrôlé par les oligarques. La tonalité de ses discours sur l'Internet est neutre ou positive. Lors de sa première conférence de presse en ligne, le président russe déclare avec regret qu'il « n'utilise pas l'Internet mais ses filles l'utilisent ». Pendant sa rencontre avec des jeunes webdesigners le 20 juin 2002, Vladimir Poutine déclare que l'Internet est « très important pour le pays et pour la jeune génération ». La deuxième période commence en décembre 2004 et se termine en mars 2008, ce qui correspond au deuxième mandat de Poutine. Ces années sont marquées par les attaques contre l'opposition politique et les oligarchies. Ces processus sont accompagnés par la 
croissance économique et l'augmentation du niveau de vie de la population. La tonalité des discours devient neutre ou négative. À notre avis, le tournant considérable se manifeste après le 6 juin 2006, avec l'échec de la première conférence de presse organisée par le groupe BBC et le moteur de recherche russe Yandex. En effet, le président russe n'a répondu à aucune question sélectionnée par les internautes. Cette conférence a montré qu'il ne maîtrise pas bien le modèle de dialogue proposé par ce dispositif. Après cette conférence, Poutine, déçu par la réaction des internautes, a caractérisé l'Internet comme «l'environnement pour les jeunes » qui sont politiquement passifs. C'était la dernière fois qu'il participait à ce type de conférence.

À partir de l'année 2006, deux dispositifs de communication politique s'établissent: la conférence de presse annuelle et l'émission intitulée Ligne directe. L'étude du choix lexical dans les discours de Poutine de cette période montre la prédominance de mots aux connotations négatives associés à l'Internet: "piratage ", "menace", "terrorisme ", «criminel», «malfaiteurs». À notre avis, Vladimir Poutine appréhende les menaces potentielles que l'Internet représente pour le pouvoir russe. Aussi continue-t-il la politique de la préservation de l'identité et de la culture russe. C'est sa réponse aux pouvoirs normalisants des flux mondiaux de l'information, à la globalisation et aux industries culturelles occidentales.

La troisième période correspond à l'élection de Dmitri Medvedev en tant que président du pays en 2008 et dure jusqu'à la campagne présidentielle en mars 2012. Vladimir Poutine devient alors Premier ministre. L'étude des discours de cette période marque un virage vers les stratégies de la disqualification d'Internet. Ce dispositif est présenté par Poutine comme une menace pour l'identité nationale et la langue russe, le moyen de propagation de la culture occidentale de «bas de gamme». De plus, le Premier ministre caractérise les réseaux numériques comme un environnement hostile, favorisant le «terrorisme », la « criminalité » et la "pédophilie ». Par exemple, dans son discours du 22 janvier 2010 devant le Conseil d'État, Vladimir Poutine a déclaré que « la moitié du contenu d'Internet est de la pornographie ». C'est à cette période que le sujet du contrôle de l'Internet apparait dans les discours des hommes politiques russes. Le résumé des trois périodes dans les discours de Vladimir Poutine sur Internet et TIC est présenté dans le tableau 3. 
Tableau 3. - Classification des discours de Vladimir Poutine sur l'Internet et les NTIC entre 2001 et 2012.

\begin{tabular}{|c|c|c|c|c|}
\hline Période & $\begin{array}{c}\text { Contexte politique et } \\
\text { économique }\end{array}$ & $\begin{array}{l}\text { Type de } \\
\text { discours }\end{array}$ & Tonalité & Termes voisins \\
\hline 2001-2004 & $\begin{array}{l}\text { Arrivée au pouvoir, médias } \\
\text { contrôlés par l'oligarchie }\end{array}$ & $\begin{array}{c}2 \text { discours } \\
\text { de type } 1 \text { et } 2\end{array}$ & $\begin{array}{c}\text { Positive ou } \\
\text { neutre }\end{array}$ & $\begin{array}{c}\text { Рост, экономика, развитие, } \\
\text { общение, коммуникации }\end{array}$ \\
\hline 2004-2008 & $\begin{array}{c}\text { Etat reprend contrôle sur les } \\
\text { chaines télévisées, images } \\
\text { du "bon tzar » et du «père } \\
\text { de la nation» }\end{array}$ & $\begin{array}{c}3 \text { discours } \\
\text { de types } 1,2 \text { et } 3\end{array}$ & $\begin{array}{l}\text { Neutre ou } \\
\text { négative }\end{array}$ & $\begin{array}{c}\text { Молодежь, времяпровождение, } \\
\text { нравственность, контроль, } \\
\text { демократия, медиа, нацпроект, } \\
\text { технологии, творчество, } \\
\text { перспектива, цензура }\end{array}$ \\
\hline 2008-2012 & $\begin{array}{c}\text { Discours de } \\
\text { «modernisation» de } \\
\text { Medvedev, } \\
\text { Mobilisation citoyenne dans } \\
\text { le contexte économique et } \\
\text { politique difficile }\end{array}$ & $\begin{array}{c}6 \text { discours } \\
\text { de type } 1 \text { et } 2\end{array}$ & Mixte & $\begin{array}{c}\text { Вызов, общество, демократия, } \\
\text { контроль, терроризм, } \\
\text { порнография, свобода, } \\
\text { криминал, защита, самозащита, } \\
\text { цензура, культура }\end{array}$ \\
\hline
\end{tabular}

aux discours de Dmitri Medvedev, ils ne peuvent pas être analysés avec la même approche diachronique. Ses idées ne changent pas et restent stables lors de son mandat. Cela peut être expliqué par la période relativement courte de sa gouvernance et sa place particulière dans les stratégies communicationnelles de l'État russe. russes sur les NTIC et l'Internet, c'est l'image de «l'ennemi», défini ou anonyme. L'abondance du lexique guerrier est considérée par Patrick Sériot comme une des figures de style propres au discours soviétique (Sériot, 1985). Comme notre analyse le montre, le vocabulaire militaire est très présent dans le corpus étudié : les termes de défense, lutte, destruction, menace. Poutine et Medvedev utilisent les images militaristes, la rhétorique de mobilisation contre l'ennemi externe ou interne. Ceci est dans le but de créer un climat émotionnel négatif autours des NTIC et l'Internet, inspirer l'anxiété, faire adhérer et mobiliser l'audience. L'identité post-soviétique est construite ici autour de l'opposition à l'Occident, la résistance à l'intrusion étrangère qui menace les "vraies " valeurs «traditionnelles » russes. Ces discours contribuent à la création d'une barrière contre l'influence de la culture occidentale corrompue qui ne correspond pas aux valeurs russes traditionnelles (МоРОзов, 2010) :

ЕСЛИ КТО-ТО ИСПОЛЬЗУЕТ ЭТИ СРЕДСТВА (СОВРЕМЕННЫЕ СРЕДСТВА МАССОВОЙ КОММУНИКАЦИИ) В НЕБЛАГОВИДНЫХ ЦЕЛЯХ - НУ ЧТО Ж, ОБЩЕСТВО ДОЛЖНО ИМЕТЬ ВНУТРЕННЮЮ СИСТЕМУ САМОЗАЩИТЫ, И У НАШЕГО НАРОДА ЭТА СИСТЕМА САМОЗАЩИТЫ ОЧЕНЬ КРЕПКАЯ, ВРЯД ЛИ КОМУ-ТО УДАСТСЯ ЕЕ РАСКАЧАТЬ 6.

Encore un élément soviétique que nous avons identifié dans le discours de Vladimir Poutine, c'est l'appréciation morale des événements qui est très importante pour l'audience russe. Les citoyens attendent du leader d'État la définition de tout ce qui est bon, ce qui n'est pas bon dans la société. Dans ces objectifs, Poutine utilise les discours "moralistes », les réflexions sur les mœurs. Les figures de style telles que des métaphores et des épithètes servent cette stratégie. Poutine possède une crédibilité suffisante afin de 
mener ces discours. Il « symbolise le retour du vrai pouvoir ». C'est un « acteur politique issu de l'ancien système, serviteur de l'État soviétique. C'est le porteur d'un habitus qui rend possible la reprise des anciens rôles" (Nikolski, 2010). Vladimir Poutine définit l'Internet comme appartenant incontestablement au monde étranger et hostile :

ТЕПЕРЬ ПО ПОВОДУ ФАЛЬСИФИКАЦИЙ И ССЫЛОК НА ИНТЕРНЕТ. ДА В ИНТЕРНЕТЕ 50 ПРОЦЕНТОВ ПОРНОМАТЕРИАЛОВ! ЧТО МЫ БУДЕМ ССЫЛАТЬСЯ НА ИНТЕРНЕТ?! В СУД НАДО ИДТИ С ДОКАЗАТЕЛЬСТВАМИ

Un nuage des mots voisins à « Internet » dans les discours publics de Vladimir Poutine est présenté dans la figure 1.

Figure 1. - Un nuage des mots voisins à « Internet » dans les discours publics de V. Poutine.

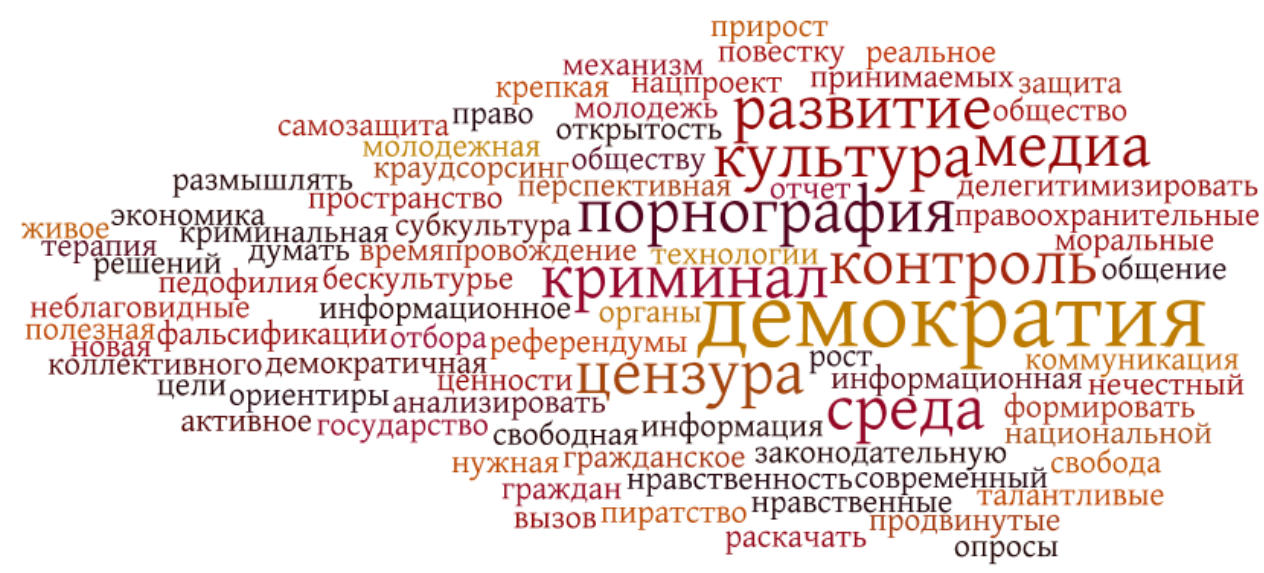

Cette figure est créée avec le logiciel en ligne Wordle.net

\section{Imaginaire de technologisme occidental dans les discours de Medvedev}

Un autre imaginaire socio-discursif que nous rencontrons dans les discours politiques sur les NTIC, c'est l'imaginaire occidental de modernité et de technologisme. Celui-ci est caractérisé par Patrick Charaudeau comme un des imaginaires types de discours politique occidental. Cet imaginaire "se définit contre un passé... l'époque présente bénéficierait d'un état de savoir supérieur qui serait le premier gage de cette modernité " (Charaudeau, 2005 : 131). L'idée principale de ce discours soutient que « la technique est censée construire les outils qui permettent de gérer de la façon la plus efficace la création et la circulation des richesses » et le développement des technologies « engendrerait le progrès social » (Charaudeau, $2005: 172$ ). Nous pouvons rencontrer des éléments de cet imaginaire dans les discours de Dmitri Medvedev et beaucoup moins dans ceux de Vladimir Poutine ${ }^{8}$.

Notre étude de la fréquence et du voisinage des mots montre que c'est le mot demokratija (« démocratie ») qui apparaît le plus souvent dans les discours liés avec les NTIC. En effet, l'imaginaire de la " démocratie numérique » est très présent chez ces hommes politiques. Les réseaux numériques sont présentés comme un outil important pour améliorer le 
fonctionnement de la démocratie (Vedel, 2003). Dans l'imaginaire de la démocratie numérique, les réseaux permettraient de résoudre les problèmes que connaît la démocratie en offrant aux citoyens de participer plus activement à la vie politique. Dans ce contexte, les discours des dirigeants russes sur la démocratie se construisent comme un sophisme, une argumentation à logique fallacieuse. En manipulant les notions des institutions démocratiques et de la liberté de parole, les dirigeants russes tentent de réduire la démocratie à l'accès aux réseaux numériques. Dans le contexte politique des années 2000 , certains acteurs politiques se sont fait refuser l'accès au champ politique. Les dirigeants russes justifiaient l'ordre social et politique établi et réduisaient la participation politique à l'accès aux dispositifs participatifs en ligne. Sous la présidence de Dmitri Medvedev de 2008 à 2012, plusieurs dispositifs numériques de débats ont été mis en ligne. Les discussions et les débats organisés par le biais de ces dispositifs sont privés de tout pouvoir de décision et servent à structurer les tensions dans la société, simuler la participation. Également, l'introduction de ces dispositifs sert à discréditer les valeurs démocratiques imposées par l'Occident :

БЛАГОТВОРНОЕ ВОЗДЕЙСТВИЕ НА РАЗВИТИЕ ДЕМОКРАТИЧЕСКИХ ИНСТИТУТОВ И ПРОЦЕДУР ОКАЗЫВАЕТ И СТРЕМИТЕЛЬНОЕ РАСШИРЕНИЕ НАЦИОНАЛЬНОГО ИНФОРМАЦИОННОГО ПРОСТРАНСТВА. НО БЕССПОРНЫМ ЛИДЕРОМ РОСТА ЯВЛЯЕТСЯ, КОНЕЧНО, ИНТЕРНЕТ: ЕГО ПОСТОЯННАЯ РОССИЙСКАЯ АУДИТОРИЯ УВЕЛИЧИЛАСЬ ЗА ЭТО ВРЕМЯ БОЛЕЕ ЧЕМ В ЧЕТЫРЕ PA3A ${ }^{9}$.

26 Nous avons observé que Medvedev reprend des éléments des discours des hommes politiques américains des années 1990. En effet, en 1999, dans un de ses discours, le viceprésident américain Al Gore popularise l'expression "autoroutes de l'information ». Il annonce le début de l'économie numérique avec l'installation de l'infrastructure numérique qui permettrait de booster l'économie et la démocratie. Dmitri Medvedev reprend l'argumentation et quelques figures de ce discours. Pour lui, l'installation des réseaux numériques de communication, des «autoroutes de l'information" contribue à "l'amélioration des communications à l'intérieur de la société", au "renforcement des liens entre les individus ${ }^{10}$ ». Un des exemples est la construction en 2010 du câble optique de $10000 \mathrm{~km}$ entre Tcheliabinsk et Khabarovsk qui permet la connexion Internet à haut débit aux régions de l'Extrême-Orient. Dans ces discours, le président exprime la volonté réformatrice de rompre avec le passé, redéfinir les rapports de forces entre le pouvoir et la société par le passage de la contrainte à l'incitation. Dans son analyse critique des discours de Medvedev sur la modernisation, le spécialiste de la sociologie de la société post-soviétique Ilia Koukouline montre comment ces discours s'appuient sur la «mémoire sentimentale » des années 1960, le « dégel politique » après la chute du culte de Staline. C'est une période rare de l'histoire russe de «l'émancipation de la société de l'État » (кукулин, 2010). L'auteur considère que l'enjeu principal de la rhétorique de modernisation de la fin des années 2000 était de séduire l'intelligentsia technique postsoviétique. Trente ans plus tôt, au milieu des années 1980, cette classe a servi de base politique de la perestroïka de Gorbatchev pour penser les nouvelles réformes politiques et économiques. Pour Koukouline, la création d'une «Silicon Valley à la russe » représente une fausse copie de l'expérience américaine car, à la différence des États-Unis, la fondation de ce pôle reste toujours une initiative étatique (кукулин, 2010).

L'imaginaire occidental technologiste se manifeste explicitement dans les discours de Medvedev concernant la création en 2010 de la zone cyrillique « russe » avec l'objectif de remplacer la zone.RU latine sur Internet. Dans ces discours, cet espace numérique national est considéré par les autorités russes comme non seulement un facteur de 
croissance économique, mais aussi comme un enjeu politique important. Medvedev explique que la création de ce «territoire » vise à échapper à la domination des ÉtatsUnis dans le domaine de la gouvernance des réseaux. Dans ce contexte, la langue anglaise et l'association ICANN, contrôle de l'allocation des adresses IP et le système des noms de domaine, sont perçues comme des instruments de cette domination (Massit-Folléa, 2012). Par ailleurs, Dmitri Medvedev ne cache pas qu'il considère la zone cyrillique de l'Internet comme un instrument de la domination politique. Lors de sa rencontre avec les blogueurs russes en avril 2011, il estime l'audience potentielle sur Internet « slave » de 300 millions de personnes et déclare qu'Internet «est un puissant instrument politique». Cette stratégie des pouvoirs russes fait suite aux débats sur la gouvernance d'Internet qui ont eu lieu aux Sommets mondiaux de l'information et de la communication à Genève en 2003 et à Genève et en Tunisie en 2005. Les représentants de la Russie, de la Chine, du Tadjikistan et de l'Ouzbékistan ont proposé aux Nations unies un code mondial de bonne conduite sur Internet.

Nous pouvons supposer que les discours de Medvedev servent à développer les propos de l'idéologème de la « démocratie souveraine » formulé par Vladimir Poutine à Munich le 10 février $2007^{11}$. Dans le cadre de cet idéologème, l'identité post-soviétique russe se base sur la priorité des intérêts étatiques par rapport aux libertés individuelles en gardant une façade "démocratique ». Dans le système de ces pensées, l'identité russe se manifeste essentiellement à travers la langue russe et les valeurs qu'elle véhicule. Cette identité néo-impériale est partagée par les Russes qui habitent à l'extérieur des frontières de la Russie contemporaine, suite à l'éclatement de l'URSS. L'Internet est considéré par les hommes politiques russes comme un lieu de luttes symboliques, qui s'inscrit dans le cadre de la politique anti-américaine et anti-occidentale. Le renforcement du contrôle de l'infrastructure numérique et le système des noms de domaine est considéré comme un point important. Le renforcement du secteur cyrillique d'Internet fait partie des enjeux politiques et stratégiques afin de rivaliser avec le secteur anglophone ${ }^{12}$ :

Я СЧИТАЮ, МЫ ДОЛЖНЫ СДЕЛАТЬ ВСЁ ОТ НАС ЗАВИСЯЩЕЕ ДЛЯ ТОГО, ЧТОБЫ МЫ МОГЛИ ДОБИТЬСЯ ПРИСВОЕНИЯ В БУДУЩЕМ ДОМЕННЫХ ИМЕН В ИНТЕРНЕТЕ НА КИРИЛЛИЦЕ. КОНЕЧНО, ЗАДАЧУ ПОДДЕРЖКИ, СОХРАНЕНИЯ УСТОЙЧИВОГО ИНТЕРЕСА К РУССКОМУ ЯЗЫКУ, РУССКОЙ ЛИТЕРАТУРЕ, РУССКИМ СРЕДСТВАМ МАССОВОЙ ИНФОРМАЦИИ Я ИНАЧЕ НЕ МОГУ ОХАРАКТЕРИЗОВАТЬ КАК ПОЛИТИЧЕСКУЮ ЗАДАЧУ ${ }^{13}$.

Un nuage des mots voisins à « Internet » dans les discours publics de Dmitri Medvedev est présenté dans la figure 2 . 


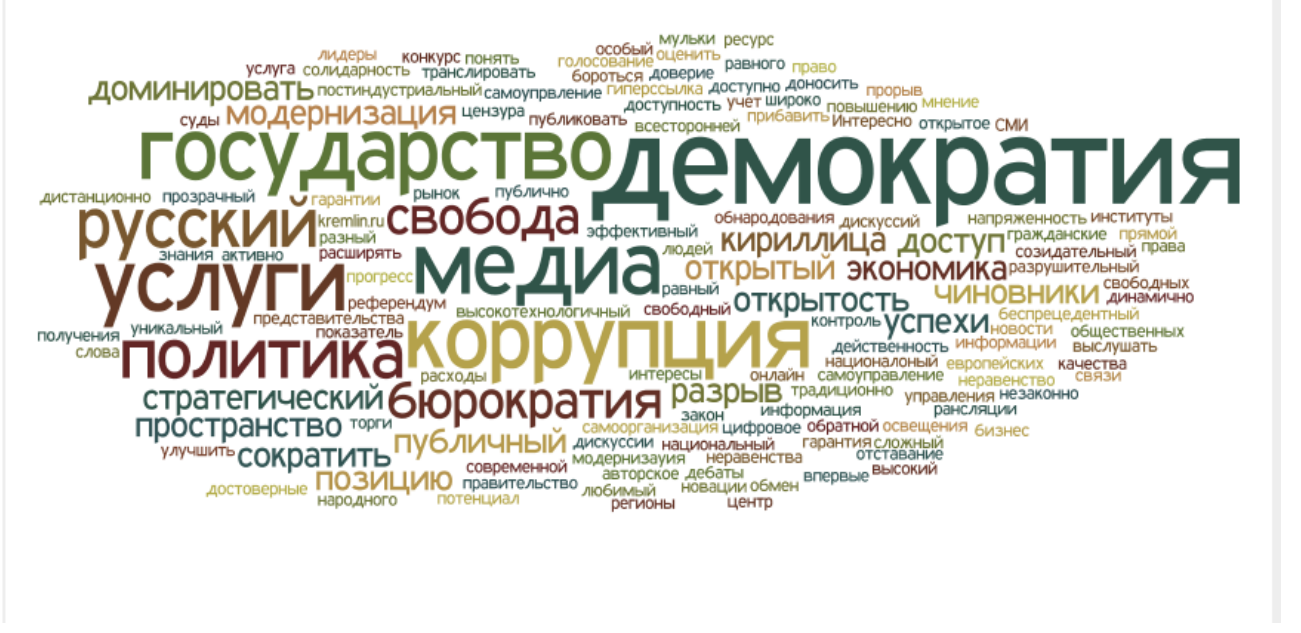

Cette figure est créée avec le logiciel en ligne Wordle.net

\section{Conclusion}

Notre analyse montre l'existence en Russie contemporaine de deux types dominants de discours politique officiel. Le premier prononcé par Vladimir Poutine se base essentiellement sur les éléments discursifs et narratifs du langage soviétique. Ce type de discours, que nous pouvons caractériser comme interne, s'adresse à la majorité de la population russe. Ces citoyens, traumatisés par la chute de l'URSS, apprécient les institutions soviétiques. Ils éprouvent beaucoup de difficultés à s'adapter à la nouvelle réalité sociale. Ces citoyens se méfient de la culture occidentale et de l'utilisation des technologies numériques qu'ils considèrent comme les éléments destructifs de l'ordre traditionnel ${ }^{14}$. Le deuxième type de discours politique, que nous considérons comme externe, est fait par Dmitri Medvedev. Ce dernier reprend les éléments de discours politiques occidentaux mais tout en mobilisant des éléments de langage libéral et moderniste pour défendre également des valeurs conservatrices. Son audience est la population éduquée des grandes villes qui a une vision critique du pouvoir postsoviétique et qui est ouverte à la culture et aux valeurs occidentales. Medvedev s'adresse également aux acteurs occidentaux, hommes politiques, journalistes afin de mettre en valeur son image de modernisateur et technocrate. Ces deux types de discours s'amalgament dans l'objectif de répondre aux attentes du public. Ils visent à séduire des électeurs, fidéliser les élites et justifier et renforcer l'ordre social post-soviétique établi.

\section{BIBLIOGRAPHIE}

BENN David (1989), Persuasion and Soviet Politics, Oxford : Basil Blackwell. 
BESANÇON Alain (1980), Court traité de soviétologie, dans Présent soviétique et passé russe, Paris :

Pluriel.

BREUILLARD Jean (1985), « Patrick Sériot, Analyse du discours politique soviétique », Revue des études slaves, $57(2), 335-341$.

CASTORIADIS Cornelius (1999), L'institution imaginaire de la société, Paris : Éditions du Seuil.

CHARAUDEAU Patrick (2005), Le discours politique. Les masques du pouvoir, Paris : Éditions du Vuibert.

DAUCÉ Françoise (2008), La Russie postsoviétique, Paris : Éditions La Découverte.

DELPORTE Christian (2009), Une histoire de la langue de bois : de Lénine à Sarkozy, Paris : Flammarion.

GoudKov Lev (2005), « Régime de Vladimir Poutine : est-il un régime autoritaire? », présentation lors de la conférence consacrée à Mikhail Khodorkovski, Moscou, <www.memo.ru> (15 novembre 2012).

HELLER Michel (1984), «Langue russe et langue soviétique », A. Bourmeyster, Novlanque, Lanque de bois et programmes narratifs. Essais sur le discours soviétique, semiologie, linguistique, analyse discursive $\left(\mathrm{n}^{\circ} 4,1-24\right)$.

INKELES Alex (1956), L'opinion publique en Russie soviétique. Une étude sur la persuasion des masses, Paris : Les Îles d'Or.

КУКУЛИН ИЛЬЯ (2010), « СЕНТИМЕНТАЛЬНАЯ ТЕХНОЛОГИЯ: ПАМЯТЬ О 1960-Х В ДИСКУССИЯХ О МОДЕРНИЗАЦИИ 2009-2010 ГОДОВ », НЕПРИКОСНОВЕННЫЙ ЗАПАС, 6(74).

MASSIT-FOLLÉA Françoise (2012), « La gouvernance de l'Internet. Une internationalisation inachevée », Le Temps des Médias, 18, 29-40.

MCAUlEy Marry (1997), Russia's Politics of Uncertainty, Cambridge : Cambridge University Press. МОРОЗОВ ВЯЧЕСЛАВ (2006), « СУВЕРЕННАЯ ДЕМОКРАТИЯ В ПОСТСУВЕРЕННОМ МИРЕ. ПУТИНСКАЯ РЕСТАВРАЦИЯ КАК ЕРАКЦИОННАЯ МОДЕРНИЗАЦИЯ », НЕПРИКОСНОВЕННЫЙ ЗАПАС, 6(50), 85-97. МОРОЗОВ ВЯЧЕСЛАВ (2010), « ПРОТЕКЦИОНИСТКАЯ МОДЕРНИЗАЦИЯ ДМИРИЯ МЕДВЕДЕВА », НЕПРИКОСНОВЕННЫЙ ЗАПАС, 6(74).

NIKOLSKI Véra (2010), « La légitimation du rôle présidentiel de Vladimir Poutine. Dispositifs de fabrication de l'image du "bon tzar" ", Réseaux, 164, 197-224.

SÉRIOT Patrick (1985), Analyse du discours politique soviétique, Paris : Édition IMSECO.

SÉRIOT Patrick (1986), « La langue de bois et son double. Une analyse des analyses du discours politique soviétique », Langage et Société, 35, 7-32.

Тном Françoise (1987), La Langue de bois, Paris : Julliard.

\section{NOTES}

1. Le chercheur français sur l'histoire de l'URSS, Laurent Coumel estime que cette expression a été employée pour la première fois en France dans le journal Le Monde le 5 juillet 1979.

2. La majorité de discours de Dmitri Medvedev sont accessibles sur le site officiel du président < www.president.ru>. Les discours de Vladimir Poutine (entre 2000 et 2008 lors de ses premiers mandats présidentiels) sont disponibles sur le site <archive.kremlin.ru>. Les discours prononcés depuis sa réélection en mars 2012 sont disponibles sur le site <www.kremlin.ru>. Un autre site < 
www.premier.gov.ru> contient l'archive des discours de Vladimir Poutine lorsqu'il occupait le poste de Premier ministre entre octobre 2008 et mars 2012. Ce site réunit les discours de Dmitri Medvedev à partir de mars 2012.

3. Les textes de lois russes doivent être publiés dans ce journal officiel. À partir du moment de la publication, ces lois entrent en vigueur. La version numérique de Rossijskaja Gazeta est disponible sur le site <www.rg.ru>.

4. Nous utilisons le signe d'astérisque pour réunir des formes des mots appartenant à la même famille. Par exemple : НовЫЕ тЕХНОЛогИи (nouvelles technologies), НОВАЯ ТЕХНолоГИЯ (nouvelle technologie), etc.

5. Discours de Vladimir Poutine devant l'Assemblée fédérale le 16 mai 2003.

6. Discours de Vladimir Poutine à la conférence avec les adhérents du parti «Russie unie » le 4 mars 2011.

7. Discours de Vladimir Poutine à la réunion du Conseil d'État sur le développement du système politique le 22 janvier 2010 .

8. Voir figure 3 de l'annexe.

9. Discours de Vladimir Poutine devant l'Assemblée fédérale le 26 avril 2007.

10. Discours de Medvedev lors de sa rencontre avec le ministre de la Communication Tschegolev le 26 avril 2010.

11. Disponible sur le site <archive.kremlin.ru> (14 décembre 2012).

12. Dans son article «Les langues sur Internet: de l'hégémonie de l'anglais au règne de la traduction », Michael Oustinoff met en doute la domination de l'anglais sur Internet. Il parle de la chute du «global english ", l'anglais planétaire. D'après les calculs de l'auteur, la part des sites rédigés en anglais a diminué de $80 \%$ en 1997 à $26,8 \%$ en 2011 . La langue chinoise occupe la $2^{\mathrm{e}}$ place $(24 \%)$ et la langue espagnole la $3^{\mathrm{e}}(7,8 \%)$. En $2011,3 \%$ des sites mondiaux sont écrits en russe.

13. Discours de Medvedev au Congrès mondial de la presse russe en juin 2008.

14. Selon le sondage réalisé en novembre 2011 par la fondation russe « Opinion Public », $55 \%$ des adultes russes n'utilisent pas Internet. Parmi ces gens, $10 \%$ n'utilisent pas Internet pour la raison de l'inaccessibilité de l'infrastructure et $45 \%$ (46 millions de personnes) perçoivent Internet comme un dispositif inutile et étranger à leur culture.

\section{RÉSUMÉS}

Malgré le démantèlement de l'URSS il y a plus de vingt ans, le soviétique est toujours fortement présent dans tous les domaines de la société russe contemporaine. Selon l'hypothèse qui nous développons dans cet article, cette persistance du soviétique se manifeste notamment par la reconstruction des éléments discursifs soviétiques dans les discours publics officiels. Notre analyse des discours des dirigeants post-soviétiques sur les nouvelles technologies de l'information et de la communication (NTIC) et l'Internet montre l'existence de deux facettes de ces discours : celle du traditionalisme imitatif présent dans les discours de Vladimir Poutine, et celle de la rhétorique de modernisation technologique véhiculée par les discours de Dmitri Medvedev. Cet article interroge également la place attribuée aux NTIC dans le discours politique des présidents russes nommés ci-dessus. 
This article studies the place of the Information and communications technology (ICT) in contemporary political discourse of the leaders of the modern Russian state. With the arrival of Vladimir Putin to power in December 1999, the Soviet spirit is to come back in contemporary Russia. According to our hypothesis, this spirit is not manifested by real restoring of the soviet institutions but by the reconstruction of "soviet" discursive elements. Our analysis shows the existence of two types of this discourse: that of "imitative traditionalism" (Vladimir Putin) and the rhetoric of "technological modernization" (Dmitry Medvedev). These discourses are two faces of the ideology of sovereign democracy. They are complementary and they legitimize the established social order.

\section{INDEX}

Mots-clés : Russie post-soviétique, Internet, NTIC, URSS, discours politique soviétique

Keywords : post-Soviet Russia, Internet, ICT, USSR, Soviet discourse

\section{AUTEUR}

\section{ALEXANDER KONDRATOV}

ATER, Laboratoire Gresec, Université Grenoble Alpes 